\title{
COPPER THIN FILM SPUTTERED ON AISI 316L FOR ANTIMICROBIAL PROPERTY
}

\author{
G. Arulkumar \\ Department of \\ Mechanical \\ Engineering, \\ Anna University, \\ Chennai, India
}

\author{
K.V.Hari Krishnan \\ Department of \\ Mechanical \\ Engineering, \\ Anna University, \\ Chennai, India
}

\author{
A. Kedharnath \\ Department of \\ Mechanical \\ Engineering, \\ Anna University, \\ Chennai, India
}

\author{
S. Kalaiselvam \\ Department of \\ Applied Science and \\ Technology, \\ Anna University, \\ Chennai, India
}

\begin{abstract}
The surgical and other biological instruments are made of Stainless Steel AISI 316L which lacks antimicrobial properties. Copper is coated on Stainless Steel substrate using DC Magnetron sputtering which is used to achieve required film of thickness $(0.5-$ $8 \mu \mathrm{m})$. The deposition pressure, substrate temperature, power supply, distance between the specimen and target are optimized and maintained constant, while the sputtering time (30-110 minutes) is varied. The sputtered copper thin film's morphology, compositional is characterized by SEM and EDAX. X-ray diffraction analysis shows copper oriented on (111) and (002) and copper oxide on (111) planes. The contact angle of copper thin film is $92^{\circ}$ while AISI316L shows $73^{\circ}$. The antimicrobial studies carried in Staphylococcus aureus, Escherichia Coli, Klebsiella pneumonia and Candida albicans shows that CFU/mL was reduced to 30 after 24 hours. The cell viability is studied by MTT assay test on Vero cell line for 24 hours, 48 hours and 72 hours and average cell viability is $43.85 \%$. The copper release from the thin film to the culture medium is $4826 \mu \mathrm{g} / \mathrm{L}$ (maximum) is estimated from AAS studies. The bacteria and fungi are found to be destroyed by the copper thin film but do not show much reaction with living Vero cells.
\end{abstract}

Keywords: Stainless Steel AISI 316L, Direct Current Magnetron sputtering, Copper thin film, Anti-microbial effect, MTT assay

\section{INTRODUCTION}

Recently many surface coating technologies have emerged to enhance the functional properties like biocompatibility and antimicrobial effect of surgical tools and medical devices. Although various methods have emerged to control the bacterial growth and contamination on medical devices and surgical tools, one of the important and highly successful method for achieving this is by surface engineering technique like thin film deposition on surgical tools to enhance their anti-microbial activity. It is a known fact that stainless steel is one of the mostly employed materials in day-to-day life. However, Stainless steel with added anti-microbial effect increases its influence in medical devices and surgical tools[1]. Even though there are many methods for modifying the surface of the stainless steel devices, sputter deposition of thin films has increased adhesion of copper layer on the metal surface and increased anti-microbial activity[2]. Copper has long been known for its anti-bacterial activity from the $5^{\text {th }}$ and $6^{\text {th }}$ millennia BC. It has long been used by man, since it was available in its native form, without the use of smelting. The spread of antibiotic resistance through selective pressure began and today has made antibiotic-resistant bacteria ubiquitous in hospitals, nursing homes, food processing plants, and animal breeding facilities. This has raised the need for different approaches to keep pathogenic microorganisms at bay. One such alternative is the use of copper surfaces in hygiene-sensitive areas [3]. The AISI316L stainless steel is coated by copper by the process of DC Magnetron Sputtering, and the structural, compositional and anti-microbial effects of the copper coated surface is studied and results are given below and these results are juxtaposed with the results obtained already due to various findings and the property of copper as an hygienic material used in hospital environments and the related topics are discussed.

\section{EXPERIMENTAL PROCEDURE:}

Sputtering is a PVD (Physical Vapour Deposition) process for depositing thin films, sputtering means ejecting material from a target and depositing it onto a substrate. The target (copper) is the source material. Substrates (stainless steel $316 \mathrm{~L}$ ) are placed in a vacuum chamber and are pumped down to a prescribed process pressure. Sputtering starts when a negative charge is applied to the target material causing a plasma or glow discharge. Positive charged gas ions generated in the plasma region are attracted to the negatively biased target plate at a very high speed. This collision creates a momentum transfer and ejects atomic size particles form the target. These particles are deposited as a thin film into the surface of the substrates. Magnetron sputtering is employed because, magnets are used to increase the percentage of electrons that take part in ionization events, increase probability of electrons striking Argon(inert gas inside the chamber), increase electron path length, so the ionization efficiency is increased significantly. Other reasons to use magnets are, Lower voltage needed to strike plasma, Controls uniformity, Increased deposition rate. Once the atoms are sputtered from the copper target, they are accelerated to high potentials in the gap between the electrodes due to applied voltage and they strike on SS 316L substrate at high velocity[1,2,4]. The growth of thin films is influenced by the type of substrate material, angle of incidence of the sputtered atoms and their surface energy. Once they get adsorbed on the SS 316L substrate, they travel continuously by random walk, on the metal surface until their energy is lost completely. Then they get deposited as thin films. This high mobility of adatoms reduces the grain boundary of the developed film because, grain boundary acts as a defect in case of thin films, and their deterioration starts at these grain boundaries. Hence sputtering is advantageous to prevent this from happening. The thickness and morphology of thin films depends upon, Gas pressure, sputtering time and current voltage and power density(see Table 1). The optimised parameters for sputtering: Vacuum base pressure: $1 * 10^{-5}$ mbar, Deposition pressure: $2 * 10^{-3}$ mbar, Substrate temperature: $200^{\circ} \mathrm{C}$, Distance between substrate target and substrate: $5 \mathrm{~cm}$, Power supply: $175 \mathrm{~W}$ (DC). 
Table 1: Variation of Coating characteristics with time of sputtering

\begin{tabular}{|c|c|c|c|}
\hline S.No. & $\begin{array}{c}\text { Time of } \\
\text { sputtering } \\
(\mathrm{min})\end{array}$ & $\begin{array}{c}\text { Coating } \\
\text { thickness } \\
(\mu \mathrm{m})\end{array}$ & $\begin{array}{c}\text { Grain size } \\
(\mathrm{nm})\end{array}$ \\
\hline 1 & 40 & $1-2$ & $110-317$ \\
\hline 2 & 50 & $2-3$ & $52-216$ \\
\hline 3 & 60 & $3-4$ & $53-146$ \\
\hline
\end{tabular}

\section{MATERIALS CHARACTERISATION}

\subsection{Morphology studies}

Typical structure of the thin film deposited by DC magnetron sputtering is a dense columnar structure. The microstructure obtained is due to the mobility of the adatoms on the surface of substrate during growth. The physical model has been proposed by A. Thornton by his SZM(Structure Zone Model)[5] which shows three typical zones on the thin films. The columnar growth is produced by low diffusion of sputter atoms, mobility of the atoms adsorbed on the substrate surface and the atomic shadow effects, which are produced by varying velocity in growth of columns and various incident angles at which adatoms arrive at the surface of substrate. These zones show a higher degree of binding among columns and at the borders of grains in columns. Initially at low time, there are lot of defects and cracks, voids which are present on the surface and the cross section view also shows the same with less column growth on the substrate, aggregation is also present between the columns. For the deposition time of $60 \mathrm{~min}$, there is decrease in voids and cracks and an significant increase in the columnar growth of the thin films and an subsequent increase in the surface area as shown in Figure1.

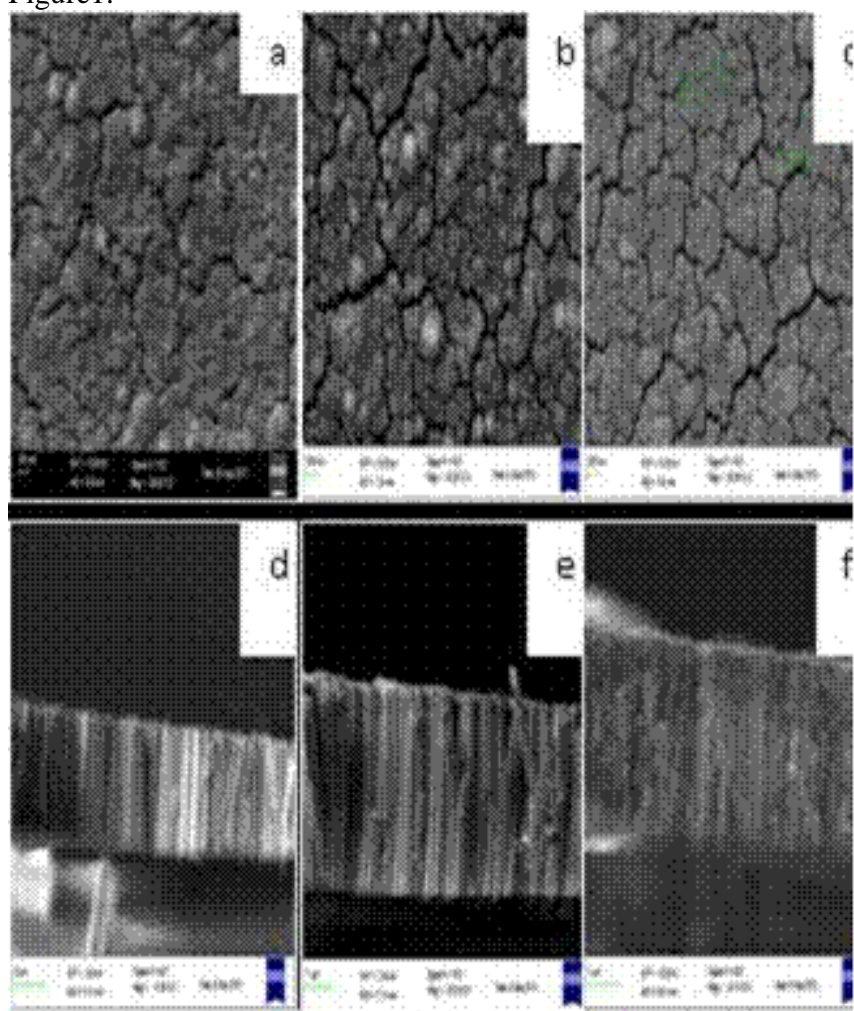

Figure 1:Scanning Electron Microscopy images of different sputter coated samples showing its morphology (a) 40

\subsection{Compositional analysis}

The images are taken from Carl Zeiss MA15 / EVO 18 which has Tungsten hairpin filament with Oxford Instruments NanoAnalysis INCA Energy 250 Microanalysis System (EDS) with $130 \mathrm{eV}$ INCAx-act Peltier cooled Analytical SDD Detector with PentaFET $®$ Precision having capability of Quantitative, Qualitative, mapping, Point \& ID etc. including cobolt standard on pin stub. The characteristic $\mathrm{X}$ rays from the samples are analyzed to find the composition of the coating. The sample has pure copper with some amount of oxygen as shown in Figure 2.

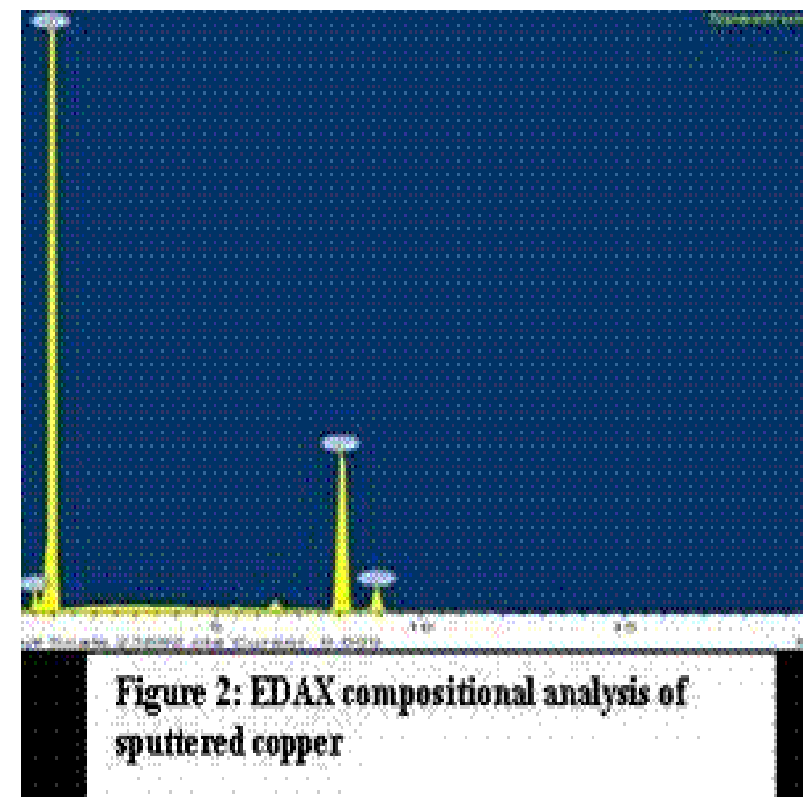

which forms $\mathrm{Cu} 2 \mathrm{O}$ which is confirmed through HR XRD. The oxidation of copper is very small so this copper oxide formed helps in the antimicrobial property and the coating will last for long time. This copper oxide release into the medium which is measured in the ppm range using AAS.

\section{3 $\mathrm{X}$ ray diffraction analysis}

High Resolution X-Ray diffraction (HRXRD) is done in PANalytical X-Pert Pro MRD Resolution $0.0001^{\circ} / 0.36$ arcsec Ge- (220) monochromator Triple axis (Xe) detector Pixel detector. As described above, the sputtered coating forms a columnar growth on the preferred planes. Copper being FCC, the

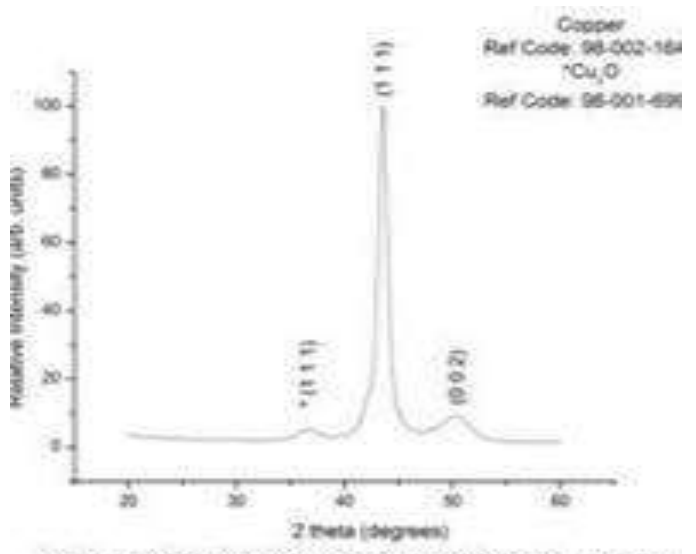

1. Figure 3: HR XTD of sputtered copper

Atoms after a random walk takes (111) and also (002) next preferred planes as shown in Figure 3. As pure copper is sputtered 
on the Stainless steel substrate, it forms oxide as the copper oxide has low Gibbs free energy than that of the pure copper. Only 13.31 atomic percent of oxygen is percent on the surface. The kinetics of the oxide is logarithmic in nature as oxidation is at room temperature. The average crystallite size is $16 \mathrm{~nm}$ with residual strain of $0.814 \%$ (cauchy contribution: 0.58377 \& gaussian contribution: 0.74106 )

\subsection{Mechanism of Copper's Antimicrobial Activity}

Copper surfaces affect bacteria in two sequential steps: the first step is a direct interaction between the surface and the bacterial outer membrane, causing the membrane to rupture. The second is related to the holes in the outer membrane, through which the cell loses vital nutrients and water, causing a general weakening of the cell. The actual activity is exhibited when holes are created in the cell membrane. It is strongly suspected that when a bacterium comes in contact with a copper surface, a short circuiting of the current in the cell membrane can occur. This weakens the membrane and creates holes. This is one way of making holes in membrane. Another is an analogy like rust weakening and making holes in a piece of metal. Now that the cell's outer envelope has been breached, there is an unopposed stream of copper ions entering the cell. This puts several vital processes inside the cell in danger. Copper literally overwhelms the inside of the cell and obstructs cell metabolism. When excess copper binds to these enzymes, their activity grinds to a halt.[6,7]. Figure 4 shows the activity of copper on various microbes.

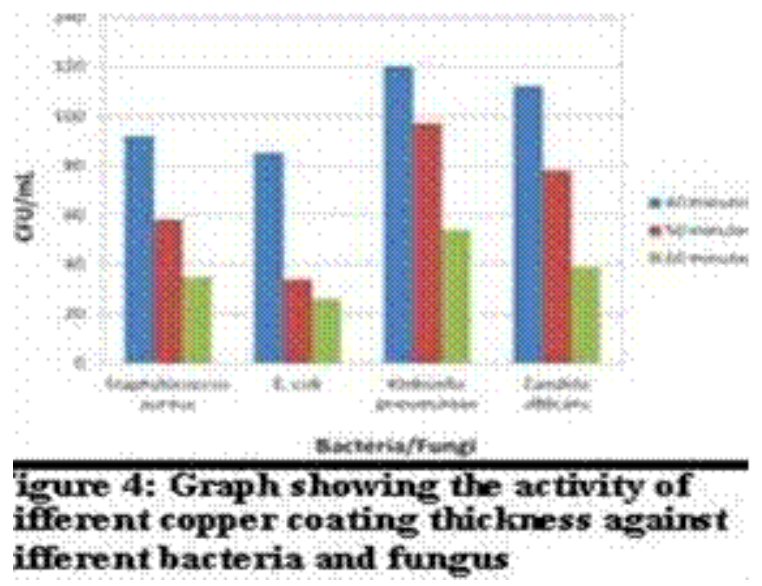

\subsection{MTT Assay}

In Vitro assay for Cytotoxicity activity (MTT assay)[8] Cells $(1 \times$ $105 /$ well) were plated in 24-well plates and incubated in 370C with 5\% $\mathrm{CO} 2$ condition. After the cell reaches the confluence, the samples were added and incubated for 24 hours. After incubation, the sample was removed from the well and washed

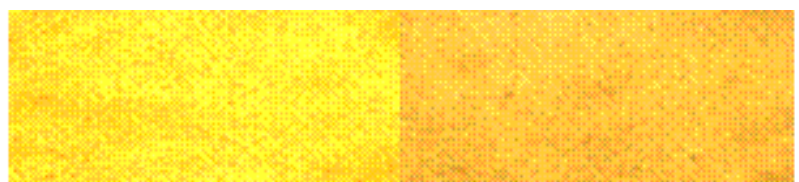

Figure 5: UV- Spectrophotometer used to find the viability of Vero Cell line. Vero cell (Left) before and (right) after

with phosphate-buffered saline ( $\mathrm{pH} 7.4)$ or MEM without serum. $100 \mu \mathrm{l} /$ well $(5 \mathrm{mg} / \mathrm{ml})$ of $0.5 \% 3$-(4,5-dimethyl-2-thiazolyl)-2,5diphenyl--tetrazolium bromide (MTT) was added and incubated for 4 hours. After incubation, $1 \mathrm{ml}$ of DMSO was added in all the

www.ijsea.com wells .The absorbance at 570nm was measured with UVSpectrophotometer using DMSO as the blank. Measurements were performed and the concentration required for a $50 \%$ inhibition (IC50) was determined graphically. The samples are compared and it is shown in Figure 5. The \% cell viability is

calculated using Eq. 1:

$\%$ cell viability $=\mathbf{A 5 7 0}$ of treated cells $/$ A570 of control cells $\times$ 100

(1)

\subsection{Copper release rate}

Sputtered coated samples of different thickness are immersed in a medium containing 90\% Dulbecco's Modified Eagle Medium (DMEM) and 10\% fetal calf serum in Carbon dioxide atmosphere. The samples for AAS are taken at an interval of 20 minutes, 40 minutes and 60 minutes. After the samples are taken few drops of Conc. Nitric acid is added to make the medium inactive. Copper release from the sputter coated sample was measured using Atomic Absorption Spectrophotometer A700 Perkin Elmer with Flame [9]. The 50 minute coating shows high release of copper from the surface in 6691 micro gram per liter when the sample is taken after 60 minutes exposure in DMEM. This is parts per billion and very small amount of copper ions are released even after one hour of exposure in DMEM. The release of copper ions for various time in the medium is shown in the Figure 6.

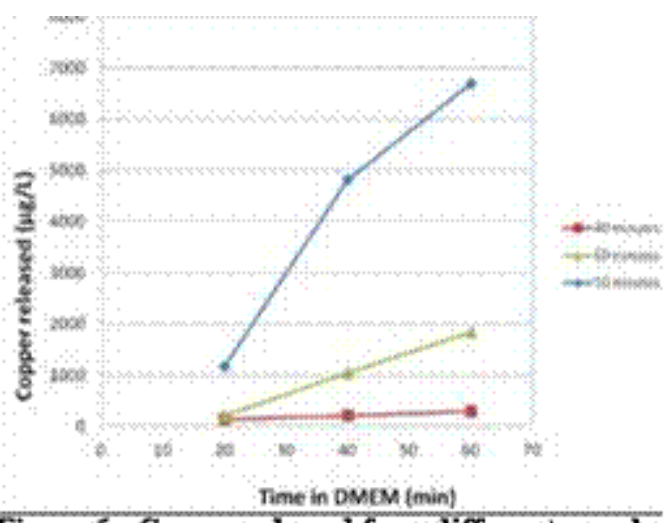

Figure 6 : Copper released from different samples at different time is found using Atomic adsorption pectroscopy

\subsection{Polarization Analysis}

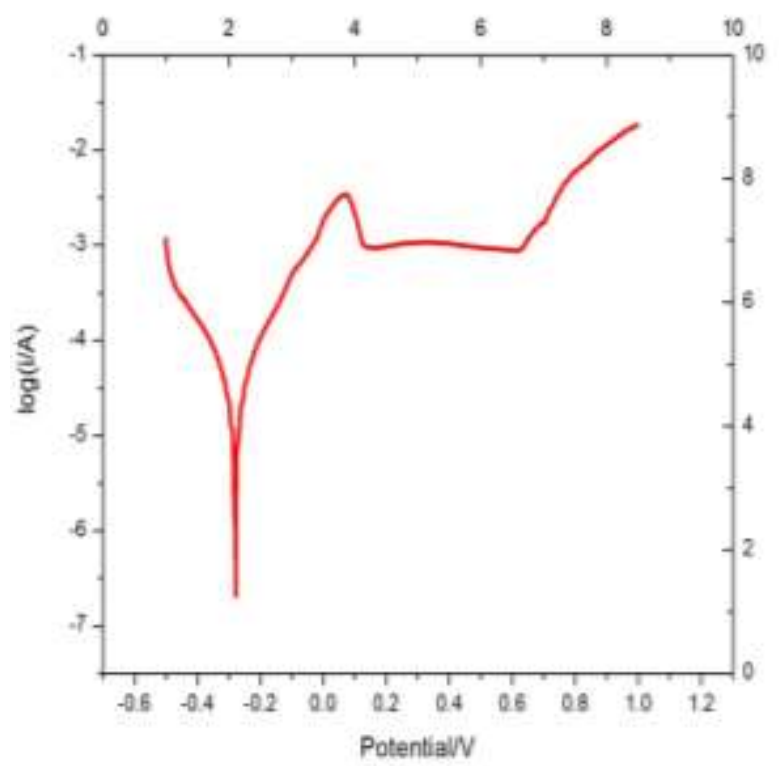

Figure 7 Tafel Plot of Copper film 
The polarization curve for the copper films measured in Simulated Body Fluid solution shows that, as the oxidation time increases, the corrosion potential increases which will reduce the thermodynamics tendency of corrosion as shown in Figure 7. The Tafel plot is plotted with $\log (\mathrm{i} / \mathrm{a}) \mathrm{Vs}$. Potential/V shows a small passivation curve and a large oxide layer curve following the passivation curve.

\subsection{Contact angle measurement}

The contact angle analysis using the Goniometer yields a value of $73^{\circ}$ and $92^{\circ}$ (maximum) for substrate and copper coated samples respectively as shown in Figure 8 . The columnar structure of the sputtered copper grains increases the contact angle and allows easy cleaning of the sample. The copper film is not hydrophobic in nature but shows a good increase in the contact angle than AISI $316 \mathrm{~L}$.

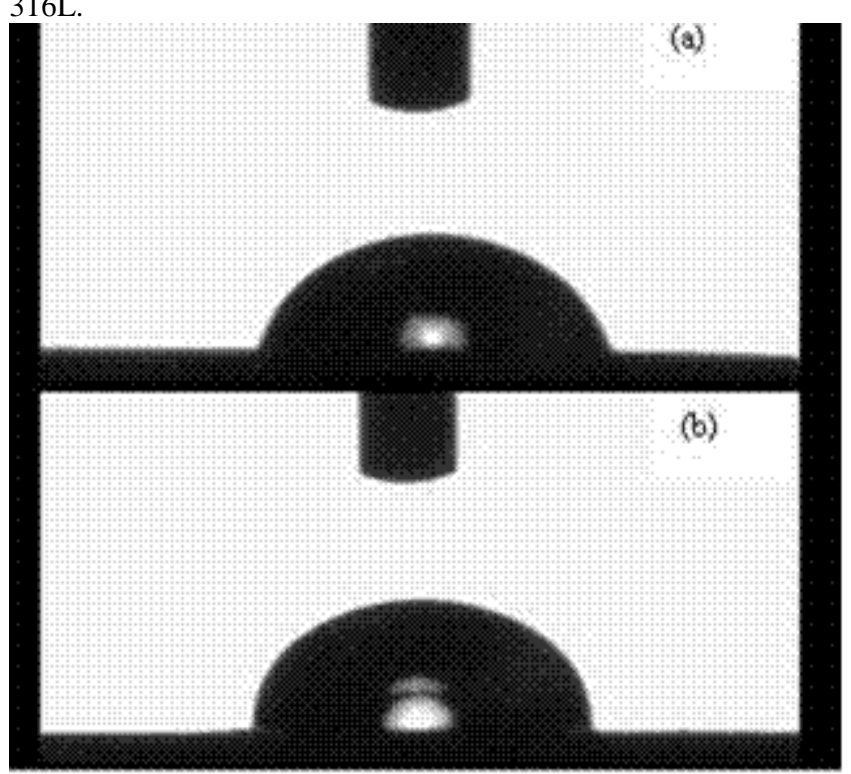

Figure 8 Contact angle measured for (a) AISI 316L, and (b) sputter coated copper film sample

\section{CONCLUSION}

The deposition of $\mathrm{Cu}$ thin film coating on AISI316L was done successfully using DC Magnetron sputtering. The structural and compositional analysis of the thin film on the substrate shows a dense columnar structure with increasing density as the sputtering time increases and it mainly contains Copper $(86.69 \%)$ and a very small amount of Oxygen (13.31\%) on the surface and it is also proven by characteristic X-ray analysis, which shows that $\mathrm{O}_{2}$ which is present, forms $\mathrm{Cu}_{2} \mathrm{O}$ shown by $\mathrm{HR}-\mathrm{XRD}$ (peak along [111]). The anti-microbial property of AISI316L has been greatly improved by the deposition of $\mathrm{Cu}$ on its surface. The biocompatibility test (MTT assay) has done on Vero cell line shows $43.85 \%$ viability after $72 \mathrm{~h}$. The deposited layer was not only stable, but also adherent to the substrate. The modified SS surfaces with its high bacterial killing rate and its durable nature make them promising candidates in hospital environments for surgical instruments and other similar applications.

\section{ACKNOWLEDGEMENTS}

This project was funded by Centre for Technology Development and Transfer, Anna University. We thank Centre for Nanoscience and Nanotechnology, Anna University for DC Magnetron sputtering. We also thank Department of Chemistry, Anna University and Department of Chemistry, Indian Institute of Technology Madras for providing various facilities.

\section{REFERENCE}

1. Xiangyu Zhang, Xiaobo Huang, Li Jiang, Yong Ma, Ailan Fan, Bin Tang. Surface microstructures and antimicrobial properties of copper plasma alloyed stainless steel, Applied Surface Science 258 (2011) 1399- 1404.

2. Borkow, G. Gabbay, J. Journal of Photochemistry and Photobiology A : Chemistry Antimicrobial $\mathrm{Cu}$ functionalized surfaces prepared by bipolar asymmetric DC-pulsed magnetron sputtering ( DCP ) Curr. Med. Chem. 3 (2009) 272

3. Casey, A. L, Role of copper in reducing hospital environment contamination. J. Hosp. Infect., 2010, 74:72-77

4. Kelly,P., Arnell, R., Magnetron sputtering: a review of recent developments and applications. Vacuum, Vol. 56, (September 1999) 159-152.

5. Thornton, J., Influence of apparatus geometry and deposition conditions on the structure and topography of thick sputtered coating, J. Vac. Sci. Technol., Vol. 11, (1974) 666-670

6. Gregor Grass,1 Christopher Rensing,2 and Marc Solioz3, Metallic Copper as an Antimicrobial Surface, Applied and Environmental Microbiology, Mar. 2011, p. $1541-1547$.

7. Dan Z G , Ni H W, Xu B F, Microstructure and Antibacterial Properties of AISI 420 Stainless Steel Implanted by Copper Ions [J]. Thin Solid Films, 2005, 492(1/2): 93.

8. Tim Mosmann, Rapid colorimetric assay for cellular growth and survival: application to proliferation and cytotoxicity assays, Journal of lmmunological Methods, 65 (1983) 55-63.

9. F. Heidenau, W. Mittelmeier, R. Detsch, M. Haenle, F. Stenzel, G. Ziegler, H.A. Gollwitzer, Analysis of the release characteristics of $\mathrm{Cu}$-treated antimicrobial implant surfaces using atomic absorption spectrometry J. Mater. Sci. Mater. Med. 16 (2005) 883. 